\title{
Error Performance Analysis of Zero Forcing Precoders for Spatial Modulation Broadcast Channels
}

\author{
Jiankang Gao ${ }^{1, a}$, Yanhui Lu ${ }^{1, b}$ \\ School of Information Engineering \\ Zhengzhou University \\ Zhengzhou, China \\ 2966326519@qq.com ${ }^{1, a}$, ieyhlu@zzu.edu.cn ${ }^{1, b}$
}

\begin{abstract}
Spatial Modulation is a newly developed concept for multi-antenna systems, which could greatly reduce the signal processing complexity and hardware implementation burden faced by these systems today. However, there are few works on the analysis of grouped Spatial Modulation, which has relatively large bit error rate(BER). In this paper, we propose a novel approach that the BER is approximatively calculated by the Chi square distribution and study the influence of the number of $\operatorname{groups}(K)$ at the sending end and the number of users $(M)$ at the receiving end on the BER in the downlink of Rayleigh channels. When the number of groups $(K)$ is increases while the number of users $(M)$ is constant, the error performance is improved obviously. Finally, Monte Carlo Simulations is provided to verify the analytical results.
\end{abstract} MIMO

Keywords—Spatial Modulation; precoders; error performance;

\section{INTRODUCTION}

Theoretical and practical results obtained during the past years have shown that Multiple-Input-Multiple-Output (MIMO) wireless systems can significantly increase the capacity of wireless networks[1-3].In particular, compared with the traditional modulation and coding schemes, Spatial Modulation(SM) achieves a higher data rate, with a lower complexity receiver[4-7]. However, existing studies on SM have mainly focused on the analysis of capacity, and there is little work on the analysis of the bit error rate(BER).

In this paper, at first, the transmit antennas of base station are grouped to improve the transmission rate and the SM concept is applied to each antenna group. Let us take the following case as an example. With 16 base station antennas and BPSK modulation in the downlink of SM broadcast channels, the system can only achieve a transmission rate of $\left(\log _{2} 16+1\right)=5$ bit; while the system can transmit $4 \times\left(\log _{2} 16 / 4\right.$ $+1)=12$ bits by evenly dividing all the antennas into 4 groups. Hence, grouped the transmit antennas can increase the transmission rate of SM. Second, we use zero forcing for the downlink of SM broadcast systems and calculate the BER by the Chi square distribution. Finally, we analyze the influence of the number of groups and the number of receiving antennas on the BER and that how to effectively reduce the BER by changing the number of groups.

\author{
Shouyi Yang 2,a ${ }^{2}$ Xiaomin $\mathrm{Mu}^{2, \mathrm{~b}}$ \\ School of Information Engineering \\ Zhengzhou University \\ Zhengzhou, China \\ iesyyang@zzu.edu.cn 2,a , iexmmu@zzu.edu.cn ${ }^{2, b}$
}

This paper is organized as follows. Section II describes the system and the model used. Section III presents the proposed the zero forcing precoders scheme. Section IV provides performance analysis and conclusion is given in Section V.

\section{SYSTEM MODEL}

The following notations are used throughout the paper: the superscripts $\mathrm{T}$ denote the transpose operations, and superscript $\mathrm{H}$ denotes the transpose conjugate; $\|\boldsymbol{A}\|$ denotes the Frobeniusnorm of $\boldsymbol{A} ; \boldsymbol{c n}(0, b)$ stands for complex Gaussian distribution with mean 0 and variance $b$.

We consider a broadcast (downlink) channel with a single base station equipped with $N_{t}$ antennas, and $M$ users each with one antenna. We evenly divided the $N_{t}$ antennas into $K$ groups and used SM in the transmit antennas of the same group. There are $N_{t} / K$ antennas in each group. The communication channel between each pair of transmit and receiving antennas is modelled by slowly time varying flat Rayleigh fading. In the analysis, we assume that channel state information is available at the base station .

In the downlink, the base station transmits independent information to each user. Let y be the received signal at user $m$. The received signal can be expressed as

$$
\boldsymbol{Y}=\left[y_{1} \ldots . y_{m}\right]^{\mathrm{T}}=\boldsymbol{H} \boldsymbol{x}+\left[n_{1} \ldots . n_{m}\right]^{\mathrm{T}}
$$

where $\boldsymbol{x}=\left[x_{1}, \ldots, x_{K}\right]$ is the transmitted signal vector with $x_{k}, k=1, \ldots, K$, being the transmitted signal from the $k$ th antenna at the base station, $\boldsymbol{n}=\left[n_{1}, \ldots, n_{M}\right]^{\mathrm{T}}$ is the noise vector with $n_{m}, m=1, \ldots, M$,being the complex noise at the $m$-th user, In the SM system, at each time instant, one antenna is activated, other antennas in the same group stay idle and assumed that it has no influence on channel. Hence, $\boldsymbol{H}$ can be expressed as a $M \times K$ MIMO channel matrix with its element $h_{m, k} \sim c n(0,1)$, denoting the complex fading channel gain from the $k$-th transmitter antenna at the base station to the $m$-th user. We assume that the channel fading coefficients of the channel matrix $\boldsymbol{H}$ are independent from each other. For high data rate transmission, the channel matrix follows a block 
fading model, which means the fading coefficients remain constant within a frame and change independently from frame to frame. The power constraint $\|\boldsymbol{x}\|^{2}=1$ is imposed on the signals. In addition, we assume that all the users have the same noise level, that is $n_{m} \sim c n\left(0, \sigma^{2}\right)$.Therefore the signal-tonoise ratio is $\gamma=1 / \sigma^{2}$.

\section{ZERO FORCING SCHEME}

In the downlink MIMO broadcast channel, let us define the information symbol vector as $\boldsymbol{u}=\left[u_{1}, \ldots, u_{m}\right]^{\mathrm{T}}$, which contains the independent information symbols of the $M$ users. Assume that $u_{m}, m=1, \ldots, M$, are drawn from the same symbol constellation with $E\left(\left|u_{m}\right|^{2}\right)=1$. The symbol vector is linearly processed by a matrix $\boldsymbol{A}$ with size $K \times M$ Considering the power constraint $\|\boldsymbol{x}\|^{2}=1$, the transmitted signal vector $X$ can be expressed as

$$
x=\frac{A \boldsymbol{u}}{\sqrt{\beta}}
$$

where $\beta$ is a power normalisation factor given by $\beta=\|\boldsymbol{A u}\|^{2}$.

The number of users that can be simultaneously supported by the base station is less than the number of base station antennas, that is $M \leq K$. The received signal $y$ can be expressed as

$$
\boldsymbol{y}=\frac{1}{\sqrt{\beta}} \boldsymbol{H A u}+\boldsymbol{n}
$$

It is obvious from (3) that $y$ can be used directly as the estimated information symbol vector $\hat{u}$, that is $\hat{u}=y$.

The precoder can be designed based on the ZF criterion[8]. The precoding matrix is given by

$$
\boldsymbol{A}=\boldsymbol{H}^{\mathrm{H}}\left(\boldsymbol{H} \boldsymbol{H}^{\mathrm{H}}\right)^{-1}
$$

Substituting (3) into (2), we get the estimate of the information symbols at the users side as

$$
\hat{\boldsymbol{u}}=\boldsymbol{y}=\frac{\boldsymbol{u}}{\sqrt{\beta}}+\boldsymbol{n}
$$

Considering $\beta=\|\boldsymbol{A u}\|^{2}=\boldsymbol{u}^{\mathrm{H}}\left(\boldsymbol{H} \boldsymbol{H}^{\mathrm{H}}\right)^{-1} \boldsymbol{u}$

We have the detection $\gamma$ of the mth user as

$$
\gamma=\frac{1}{\sigma^{2} \beta}=\frac{1}{\sigma^{2} \boldsymbol{u}^{\mathrm{H}}\left(\boldsymbol{H} \boldsymbol{H}^{\mathrm{H}}\right)^{-1} \boldsymbol{u}}
$$

If we assume the entries of $u$ are standard Gaussian variables, that is,$u_{m} \sim c n(0,1)$, then the power normalisation factor $\beta=\|\boldsymbol{A u}\|^{2}=\boldsymbol{u}^{\mathrm{H}}\left(\boldsymbol{H} \boldsymbol{H}^{\mathrm{H}}\right)^{-1} \boldsymbol{u}$ in the downlink has a scaled $F$-distribution with the parameters $n_{1}=2 M$ and $n_{2}=2(K-M+1)$, which can be represented by

$$
\beta \sim \frac{M}{K-M+1} F_{n_{1}, n_{2}}
$$

The probability density function (pdf ) of $b$ can be expressed as

$$
f(\beta)=\frac{K !}{(M-1) !(K-M) !(1+\beta)^{K+1}}
$$

As we can see from (6), the detection $\gamma$ is an scaled inverse of the normalisation factor $\beta$ that has a scaled $F$ distribution. It is shown in [9] that the inverse of an $F$ variable with parameters $n_{1}$ and $n_{2}$, denoted by $F_{n_{1}, n_{2}}$, is also an $F$ variable but with parameters $n_{2}$ and $n_{1}$. Therefore $\gamma$ also has a scaled $F$-distribution represented by

$$
\gamma \sim \frac{K-M+1}{\sigma^{2} M} F_{2(K-M+1), 2 M}
$$

Its probability density function is given by

$$
f(\gamma)=\frac{\left(\sigma^{2} \gamma\right)^{K-M}}{\left(1+\sigma^{2} \gamma\right)^{K+1}} \frac{\sigma^{2} K !}{(M-1) !(K-M) !}
$$

\section{PERformance Anslysis}

\section{A. Theoretical calculation of BER}

let us consider that the $F$-distribution with parameter $n_{1}$ and $n_{2}$ can be accurately approximated by a Chi square distribution with degree of freedom $n_{1}$, denoted by $G\left(\lambda n_{1} x\right)$, where $\lambda$ is a shrinking factor[10] and $n_{2} / n_{1} \geq 3$. That is

$$
\begin{aligned}
& F_{n_{1}, n_{2}}(x) \sim G\left(\lambda n_{1} x\right) \\
& \lambda=\frac{n_{1}+2 n_{2}-2+n_{1} x / 3}{2 n_{2}+4 n_{1} x / 3}
\end{aligned}
$$

We can get an approximation of the density of $\lambda$ as

$$
f(\gamma) \cong g\left(\lambda n_{1} \gamma / a, n_{1}\right) \frac{d\left(\lambda n_{1} \gamma / a\right)}{d \gamma}
$$

where $a=(K-M+1) / \sigma^{2} M$ equals the scale factor in the scaled $F$-distribution, $g\left(\lambda n_{1} \gamma / a, n_{1}\right)$ is the density of the Chi square distribution with degree of freedom $n_{1}$, given by

$$
g\left(\lambda n_{1} \gamma / a, n_{1}\right)=\frac{\left(\lambda n_{1} \gamma / a\right)^{n_{1} / 2-1} \exp \left(-\left(\lambda n_{1} \gamma / a\right) / 2\right)}{2^{n_{1} / 2} \Gamma\left(n_{1} / 2\right)}
$$

With $\Gamma(n)$ denoting the Gamma function, $\Gamma(n)=(n-1)$ !. 


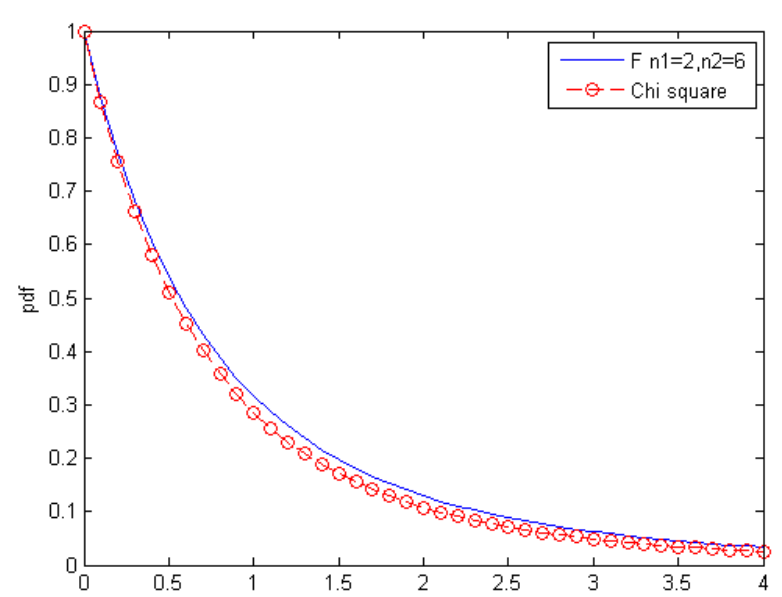

Fig.1 Comparison between the F-distribution and the approximation using shrinking Chi-square distribution $\left(n_{1}=2, n_{2}=6\right)$

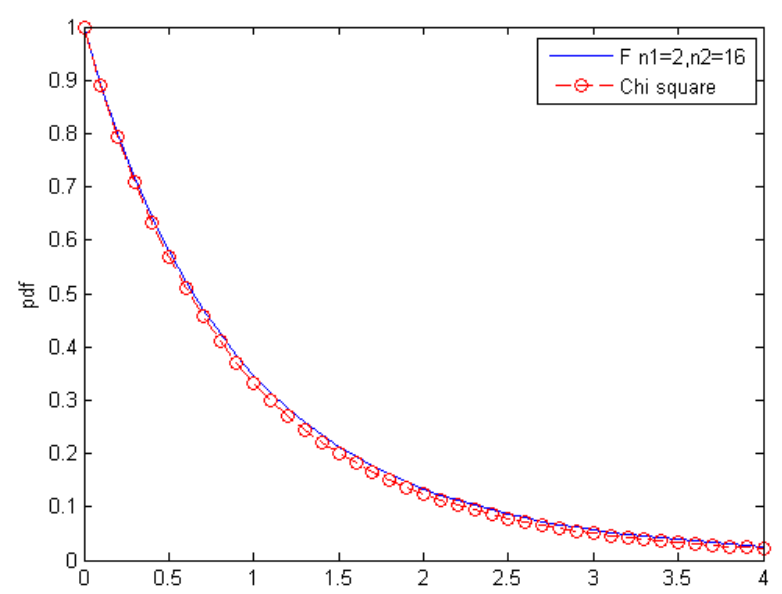

Fig.2 Comparison between the F-distribution and the approximation using shrinking Chi-square distribution $\left(n_{1}=2, n_{2}=16\right)$

Fig. 1 and Fig.2 compares the approximate density of $\gamma$ with the original scaled $F$-distribution. We can see that the approximation is very accurate when $n_{2} / n_{1} \geq 3$. The higher the ratio ,the more accurate approximation.

From (12), the $\lambda$ can be simplified as follows

When $x \gg 1,\left[\lambda\left(x ; n_{1}, n_{2}\right) n_{1} x\right] \sim n_{1} x / 4$

When $n_{2} \gg 1,\left[\lambda\left(x ; n_{1}, n_{2}\right) n_{1} x\right] \sim n_{1} x$

The upper bound of the BER of the zero forcing can be computed as [11]

$$
\begin{aligned}
& p_{e} \leq N_{e} E\left[Q\left(\sqrt{c^{2} \gamma / 2}\right)\right] \\
& =N_{e} \int_{0}^{+\infty} Q\left(\sqrt{c^{2} \gamma / 2}\right) f(\gamma) d \gamma
\end{aligned}
$$

where $c^{2}$ is the minimum squared Euclidean distance in the modulation constellation, $N_{e}$ is the average number of nearest neighbors with the minimum squared Euclidean distance in the constellation, and $Q(x)$ is the $Q$ function. To obtain the BER, we consider a (15) and (16) respectively.

$$
\lambda=1 / 4, F_{n_{1}, n_{2}}(x) \sim G\left(n_{1} / 4 x\right)
$$

We can get an approximation of the density of $\gamma$ as

$$
\begin{aligned}
& f(\gamma) \simeq g\left(n_{1} \gamma / 4 a, n_{1}\right) \frac{d\left(n_{1} \gamma / 4 a\right)}{d \gamma} \\
& =\frac{1}{4} \frac{\sigma^{2} M^{K-M+1}}{(K-M) !} e^{-K \sigma^{2} \gamma}\left(\sigma^{2} \gamma / 4\right)^{K-M}
\end{aligned}
$$

Substituting (19) into (17), the BER can be derived as follows

$$
\begin{aligned}
& p_{e} \simeq(1 / 4)^{K-M+1} N_{e} \frac{\sigma^{2} M^{K-M+1}}{(K-M) !} \\
& \times \int_{0}^{+\infty} e^{-K \sigma^{2} \gamma}\left(\sigma^{2} \gamma\right)^{K-M} Q\left(\sqrt{c^{2} \gamma / 2}\right) d \gamma
\end{aligned}
$$

Using the formula $\mathrm{Q}$ function

$$
\int_{0}^{+\infty} x^{2 n-1} e^{-x^{2} / 2} Q(x / \sigma) d x
$$$$
=\frac{(n-1) !}{2}\left[1-\left(1+\sigma^{2}\right)^{-1 / 2}\right]^{n} \sum_{i=0}^{n-1} 2^{-i}\left(\begin{array}{l}
n-1+i \\
i
\end{array}\right)\left[1+\left(1+\sigma^{2}\right)^{i}\right.
$$

Substituting (21) into (20), the BER can be simplified as follows

$$
\begin{aligned}
& p_{e}=\frac{N_{e}}{2^{3(K-M+1)}}\left[1-\left(1+4 M \frac{\sigma^{2}}{c^{2}}\right)^{-1 / 2}\right]^{K-M+1} \\
& \times \sum_{0}^{K-M}\left[2^{-i}\left({ }_{i}^{K-M+i}\right)\left(1+\left(1+4 M \frac{\sigma^{2}}{c^{2}}\right)^{-1 / 2}\right)^{i}\right]
\end{aligned}
$$

Similarly, we can get that as follows

$$
\begin{aligned}
& \lambda=1, F_{n_{1}, n_{2}}(x) \sim G\left(n_{1} x\right) \\
& p_{e}=\frac{N_{e}}{2^{K-M+1}}\left[1-\left(1+4 M \frac{\sigma^{2}}{c^{2}}\right)^{-1 / 2}\right]^{K-M+1} \\
& \times \sum_{0}^{K-M}\left[2^{-i}\left({ }_{i}^{K-M+i}\right)\left(1+\left(1+4 M \frac{\sigma^{2}}{c^{2}}\right)^{-1 / 2}\right)^{i}\right]
\end{aligned}
$$

To get the asymptotic error performance, we apply Taylor series approximation, that is, $x^{-1 / 2} \simeq 3 / 2-x / 2$, and $\gamma=1 / \sigma^{2} \gg 1$.In this case, we can see that the BER in (24) can be further approximated as

$$
\begin{aligned}
& p_{e} \simeq \frac{N_{e}}{2^{K-M+1}}\left(\frac{2 M}{c^{2}}\right)^{K-M+1}(1 / \gamma)^{K-M+1} \sum_{i=0}^{K-M} 2^{-i}\left(\begin{array}{l}
K-M+i \\
i
\end{array} 2^{i}\right. \\
& =\left[N_{e}\left(M / c^{2}\right)^{K-M+1} \sum_{i=0}^{K-M} \frac{(K-M+i) !}{i !(K-M) !}\right](1 / \gamma)^{K-M+1}
\end{aligned}
$$

\section{B. Simulation Results}

The following notations are used throughout the pictures below: asyb denote asymptotic bound; appr denote 
approximation; simu denote simulation. QPSK modulation scheme is employed in two pictures.

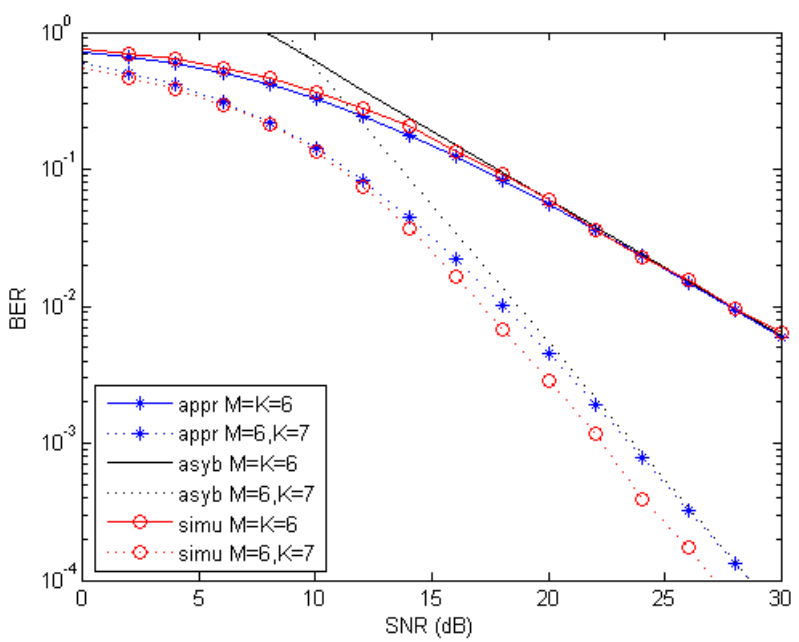

\section{Fig.3 Comparison between the $\gamma$ and the BER}

From Fig. 3, we can see that (24) is a tight upper bound to the BER of the receiver in downlink with shared power constraint and it is also a good approximation to the BER of the simulation results in downlink. when $\gamma \gg 1$, approximation is very near to the asymptotic bound.the approximation of the group with $M=K=6$ very better than the other group, because the ratio of $n_{2} / n_{1}$ is even greater, where $n_{1}=2(K-M+1)$, $n_{2}=2 M$. When the number of groups $(K)$ is increased and the number of users $(M)$ is constant, the error performance is improved obviously.

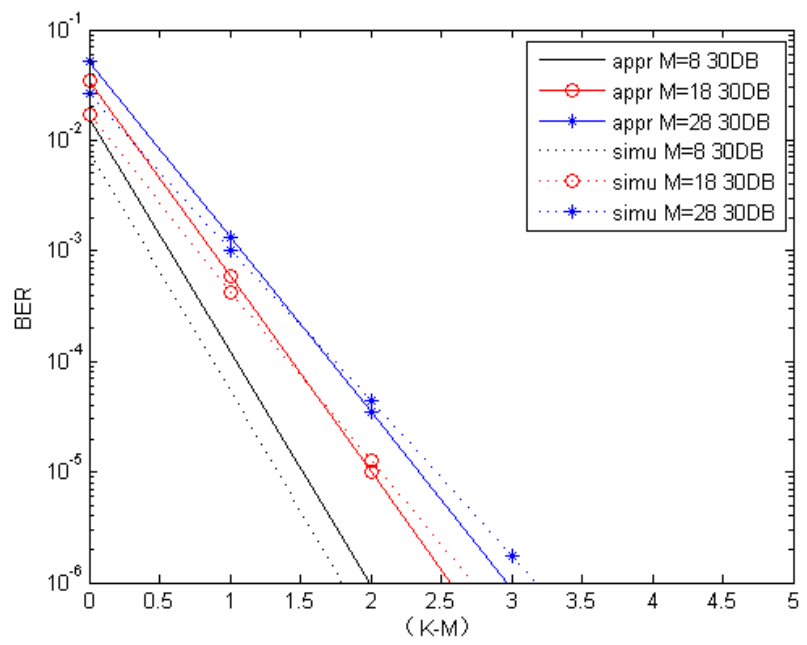

Fig.4 Comparison between the value of $K$ minus $M$ and the $\operatorname{BER}(\gamma=30(D B))$

In Fig.4,X-axis is the value of $K$ minus $M$. Y-axis is BER. When the value of $K$ minus $M$ is increased, the error performance is reduced obviously. When the value of $K$ minus $M$ increased to 1 while the number of users $(M)$ is 8 , the BER is dropped from $10^{-2}$ down to $10^{-4}$ rapidly. Nonetheless, $K$ must meet the following conditions for theoretical arithmetic: $K \leq(4 M-3) / 3$ and $K$ is an integer. In a real environmen, the transmit power will increase with $K$ increased. Therefore, there is a limit to the $K$. According to the needs of the real environment, we can get a good error performance by modulating the parameters of $K$.

\section{CONCLUSION}

In this paper, We use the Chi square distribution to calculate the BER of Spatial Modulation and study the influence of the number of groups and the number of receiving antennas on the BER. It is demonstrated that the approximation is very near to the simulation results. In addition, We can significantly reduce the BER by modulating the parameters of $K$. When $K$ is increased by 1 , the BER can be reduced by two orders of magnitude almost.

\section{ACKNOWLEDGMENT}

The work presented in this paper was supported in part by the Program for New Century Excellent Talents in University under Grant No.NCET-12-0699, National Natural Science Foundation of China under Grant No.61271421.

\section{REFERENCES}

[1] J. Mietzner, R. Schober, L. Lampe, W. H. Gerstacker, and P. A. Hoeher, "Multiple-antenna techniques for wireless communications - A compre-hensive literature survey”, IEEE Commun. Surveys Tuts., vol. 11, no. 2,pp. 87-105, 2nd quarter 2009.

[2] H. Huang, C. B. Papadias, and S. Venkatesan, MIMO Communication for Cellular Networks, Springer, Nov. 2011.

[3] M. Di Renzo, H. Haas, A. Ghrayeb, S. Sugiura, and L. Hanzo, "Spatial modulation for generalized MIMO: Challenges, opportunities and implementation”, IEEE Proc. of the IEEE, vol. 102, no. 1, pp. 56-103, Jan.2014.

[4] M. Di Renzo and H. Haas, "Performance analysis of spatial modulation," in Communications and Networking in China (CHINACOM), 2010 5th International ICST Conference on, 2010, pp. 1-7.

[5] R. Mesleh, H. Haas, S. Sinanovic, C. W. Ahn, and S. Yun, "Spatial modulation,” Vehicular Technology, IEEE Trans-actions on, vol. 57, no. 4, pp. 2228-2241, 2008.

[6] J. Jeganathan, A. Ghrayeb, and L. Szczecinski, "Spatial modulation: optimal detection and performance analy-sis,” Communications Letters, IEEE, vol. 12, no. 8, pp.545-547, 2008.

[7] S.Narayanan,M.Di Renzo,F.Graziosi, and H. Haas, “Distributed spatial modulation for relay networks”, IEEE Veh. Technol.Conf., pp. 1-5,Sep. 2013.

[8] Peel, C., Hochwald, B., and Swindlehurst, A.: 'A vector-perturbation technique for near-capacity multiantenna multiuser communication-part I: channel inversion and regularization', IEEE Trans. Comm., 2005, 53, (1), pp. 195-202

[9] Hochwald, B., and Vishwanath, S.:'Space-time multiple access:linear growth in the sumrate'. Proc. 40th Annual Allerton Conf. Communications, Control, and Computing, Allerton, IL,October 2002

[10] Li,B.,and Martin, E.:'An approximation to the F distribution using the Chi-square distribution’,Comput.Stat.DataAnal., 2002, 40,(1), pp.21-26

[11] Proakis,J.:'Digital communications’(Mcgraw Hill, New York,2008,5rd edn.) 